\title{
Risk of Phthalate Exposure among Hospitalized Patient via Intravenous Fluids Receiving
}

\author{
Forouz Rastegari ${ }^{1}$, Mohammad Mehdi Amin ${ }^{2}$, Karim Ebrahim ${ }^{2}$
}

Received: 02.11.2016

Accepted: 24.12 .2016

\section{ABSTRACT}

Background: Phthalates are widely used as plasticizers in polyethylene terephthalate (PET) products. Several pharmaceutical products have been stored in PET containers, and due to serious health effects of phthalates, migration levels of them into pharmaceutical products must be determined. In the present study, leaching levels of four common phthalate esters including di-nbutyl phthalate (DBP), diethyl phthalate (DEP), benzyl butyl phthalate (BBP) and Di-(2-ethylhexyl) phthalate (DEHP) into various types of infusion fluids form four different commercial brand were investigated.

Methods: Trace levels of phthalate esters were successfully extracted by a dispersive liquidliquid microextraction (DLLME) method using acetonitrile as dispersive and $\mathrm{CCL}_{4}$ as an extraction solvent and analyzed by gas chromatography-mass spectrometry (GC-MS).

Results: Different levels DEHP were detected in all samples (mean=10.55 \pm 6.88 and maximum=19.7 ppb). While no levels of other phthalates were detected in some samples, some other contained up to $25.1 \pm 17.7,15.4 \pm 8.3,8.1 \mathrm{ppb}$ DEP, DBP, and BBP respectively. Mean total phthalate ester (TPE) levels in infusion fluids were $7.92 \pm 5.68 \mathrm{ppb}$. Phthalate leaching into normal saline was significantly lower than other types of serums and brand ID\#3' products contain minimum phthalate levels compared to other brands.

Conclusions: Hospitalized patient particularly those who regularly take serum-therapy and children are at significant risk of phthalate exposure via intravenous fluids receiving.

Keywords: Exposure Assessment, Hospitalized Patients, Intravenous Fluids, Phthalate Esters, Toxicity.

\section{IJT 2017 (3): 33-38}

\section{INTRODUCTION}

Phthalates includingdialkyl or alkyl/aryl esters of phthalic acid (1,2-benzenedicarboxylic acid) are primarily used in polyethylene terephthalate (PET) products as plasticizers to impart flexibility and durability [1]. Globally, more than 18 billion pounds of phthalates are produced and used each year [2]. They have been used for a variety of purposes, including building materials, food packaging, clothing, toys, personal-care products (e.g. perfumes, lotions, and cosmetics), paints and industrial plastics [3].

There are several types of phthalates, but din-butyl phthalate (DBP), diethyl phthalate (DEP), benzyl butyl phthalate (BBP) and Di-(2ethylhexyl) phthalate (DEHP) are the most common. Di-(2-ethylhexyl) phthalate (DEHP) is the most commercially used plasticizer in PVC medical devices, such as IV and blood bags, typically contain $10-40 \%$ DEHP by weight; other devices, such as medical tubing may contain as much as $80 \%$ DEHP by weight [4].

Phthalates do not covalently bound to the plastic matrix and leach out from polymer when they come in contact with certain medias [5]. The major factors determining the degree of phthalates leaches to in-contact medias are the chemical properties of media, temperature, amount of phthalates in the product, agitation of the product and storage time [6].

Phthalates are toxic for several body systems. Toxicological profiles and potency vary by specific phthalate. Animal experiments have shown that the liver and reproductive systems are main target organs phthalate toxicity. Recently a great deal of concern has been raised on the

1. MSc of Chemical Toxicology, Environment Research Center, Research Institute for Primordial Prevention of Noncommunicable Disease, Isfahan University of Medical Sciences, Isfahan, Iran.

2. Department of Environmental Health Engineering, Isfahan University of Medical Sciences, Isfahan, Iran.

*Corresponding Author: E-mail: k.najafabady@ sbmu.ac.ir 
carcinogenic effects of phthalates, and some phthalates are a carcinogen in rodents. Metabolic products of phthalates have developmental, and reproductive toxicity is affecting particularly male reproductive system $[7,8]$. Some of the phthalates are an endocrine disruptor and interfere with the function of the endocrine system, responsible for growth, sexual development, and many other essential physiological functions both in males and females $[9,10]$.

Recently medical and pharmaceutical industries widely use phthalate esters for the production of various medical devices such as blood bags, intravenous fluid bags, infusion sets and pharmaceutical products containers. Infusion fluids such as normal saline, ringer's lactate, and injection water are the most well-known pharmaceutical products that stored in polyethylene terephthalate (PET) containers (Usually for several months) and directly injected into the blood stream of patients, so bioavailability of any contamination in these Infusion fluids is $100 \%$.

Although the overall benefits of medical procedures using infusion fluids outweigh the risk associated with exposure to phthalate esters, more research is needed to determine whether patients undergoing medical procedures using phthalatescontaining devices are at a higher risk for altered health outcome than the general population. There is no comprehensive study on phthalates lichgate from the plastic container to infusion fluids in Iran.

Therefore, the present study was conducted to investigate this issue in various infusion fluids from different commercial brands in Iran.

\section{MATERIALS AND METHODS}

\section{Materials}

Analytical standards of dimethyl phthalate (DMP), diethyl phthalate (DEP), bis (2ethylhexyl) phthalate (DEHP) and benzyl butylphthalate (BBP) were purchased from SigmaAldrich (Sigma, St. Louis, USA, catalog number: 41320, 53008, 36735, 36927 respectively).

The GC grade acetonitrile, carbon tetrachloride, methanol and pure water were purchased from Merck (Darmstadt, Germany).

\section{Infusion Fluids Samples}

Four commercial brands (brand ID \#) of infusion fluids were randomly purchased in the summer of 2015 from different drugstores in the Isfahan-Iran. At least three samples from different types of infusion fluids including normal saline (sodium chloride $0.9 \%$ ), ringer's lactate, dextrose and injection water were provided. All samples were labeled and stored at $4{ }^{\circ} \mathrm{C}$ in the dark until analysis. All samples were produced in spring 2015.

\section{Standard Preparations}

A mixed stock standard solution of 1000 ppm from all phthalate esters was prepared in methanol. The working standard solutions of 50, $10,5,1$ and $0.1 \mathrm{ppb}$ were prepared in ultrapure water. The stock and working standard solutions were stored at $4{ }^{\circ} \mathrm{C}$.

\section{Extraction of Phthalate}

Phthalate esters were extracted from infusion fluids by a dispersive liquid-liquid extraction (DLLME) method [11] with some modifications. An aliquot of $5.00 \mathrm{~mL}$ of each sample was placed in a $10 \mathrm{~mL}$ glass test tube with a conical bottom. A mixture of acetonitrile $(0.75$ $\mathrm{mL})$ as a dispersive solvent, and $\mathrm{CCl}_{4}(50 \mu \mathrm{L})$ as extraction solvent were used. The solvents were injected rapidly into the sample solution using $1.00 \mathrm{~mL}$ Hamilton syringe. After that, a cloudy solution (sample+acetonitrile/CCl 4 ) was formed and the analyses were extracted into the fine $\mathrm{CCl}_{4}$ droplets. After centrifugation for $5 \mathrm{~min}$ at 4500 $\mathrm{rpm}$, extraction solvent was sedimented in the bottom of the conical test tube (about $40 \mu \mathrm{L}$ ). Two microliters of sedimented phase were removed using a $10 \mu \mathrm{L}$ GC/MS microsyringe and injected into the GC system for analysis.

\section{Analytical Methods}

The extracts were analyzed by gas chromatography/mass spectrophotometry using a quadrupole Agilent GC-MS (Agilent Technologies, Palo Alto, CA, USA) model 6890N coupled to a mass selective detector model 5973 inert, operated in the electron-impact mode at 70 $\mathrm{eV}$. Data recording and instrument control were performed by the MSD ChemStation software (G1701CA; Version C.00.00; Agilent Technologies). Helium (99.999\%) was employed as carrier gas at the flow rate of $1 \mathrm{~mL} / \mathrm{min}$. The analytes were separated using a capillary column (HP-5, $30 \mathrm{~m} \times 0.25 \mathrm{~mm}$ id., $0.25 \mu \mathrm{m}$ coating thickness). The gas chromatographic conditions were as follows: Injection volume: $2 \mu \mathrm{L}$, split 
ratio: $1 / 10$, injector temperature: $280{ }^{\circ} \mathrm{C}$, The oven temperature was programmed from $100{ }^{\circ} \mathrm{C}$ (holding time $2 \mathrm{~min}$ ) to $210{ }^{\circ} \mathrm{C}$ at $10{ }^{\circ} \mathrm{C} / \mathrm{min}$ then to $250{ }^{\circ} \mathrm{C}$ at $5{ }^{\circ} \mathrm{C} / \mathrm{min}$ and finally to $280{ }^{\circ} \mathrm{C}$ at 30 ${ }^{\circ} \mathrm{C} / \mathrm{min}$ keeping the final temperature for $4 \mathrm{~min}$. The MS transfer line and ion source were kept at $290{ }^{\circ} \mathrm{C}$. The MS was tuned to $\mathrm{m} / \mathrm{z} 69,219$ and 502 for the EI corresponding to perfluorotributylamine (PFTBA). Data acquisition was carried out in the full-scan mode $(\mathrm{m} / \mathrm{z}=149)$ mode and results were qualified by comparison with the NIST and Wiley's library spectral data bank (G1035B; Rev D.02.00; Agilent Technologies).

\section{Validation of the Method}

The validation was done according to International Conference on Harmonization (ICH) recommendations for linearity, range, accuracy, and precision, limit of detection (LOD), limit of quantification (LOQ) and relative recovery [12].

\section{Statistical Analysis}

Experiments were repeated at least three times, and the results were expressed as mean \pm SD. Data were analyzed by Student $t$-test and analysis of variance (ANOVA) with significance level defined as $P<0.05$ using GraphPad-Prism 5 software (GraphPad-Prism Software Inc., San Diego, USA).

\section{Ethics}

This study was approved by the Ethics Committee of Research Institute for Primordial Prevention of Non-communicable Disease, Isfahan University of Medical Sciences, Isfahan, Iran (ID:294179).

\section{RESULTS}

\section{Validation of the Method}

This is a highly sensitive, selective and accurate analytical method for phthalate detection and determination in aqueous solutions. A good resolution was achieved for phthalates separation in this method. All four phthalate esters left the column at 11.4, 14.3, 15.4, 20.6 min (Figure 1).

Quantification was done using the external calibration method showing linear correlations with $\mathrm{R}^{2}>0.98$ for all the target analytes from the range of 1 to $100 \mathrm{ppb}$. Other method validation parameters are presented in Table1.

Table1. Method validation parameters for determination of phthalate esters by DLLME extraction and GC/MS analysis.

\begin{tabular}{|c|c|c|c|c|c|c|c|c|c|c|c|c|}
\hline Parameter & \multicolumn{4}{|c|}{$\mathrm{R}^{2}$} & \multicolumn{4}{|c|}{ LOD } & \multicolumn{4}{|c|}{ LOQ } \\
\hline Phthalates & DMP & DBP & DEHP & BBP & DMP & DBP & DEHP & $\mathrm{BBP}$ & DMP & DBP & DEHP & $\mathrm{BBP}$ \\
\hline Value & 0.99 & 0.99 & 0.99 & 0.98 & 0.23 & 0.26 & 0.107 & 0.31 & 0.78 & 0.89 & 0.36 & 1.06 \\
\hline Parameter & \multicolumn{8}{|c|}{ In-day } & \multicolumn{4}{|c|}{ Recovery\% } \\
\hline Phtha & DMP & DBP & DEHP & BBP & DMP & DBP & DEHP & BBP & DMP & DBP & DEHP & BBP \\
\hline Value & 6.3 & 6.8 & 4.5 & 7.2 & 7.6 & 6.6 & 6.5 & 9.7 & 96.1 & 88.7 & 91.2 & 87.6 \\
\hline
\end{tabular}

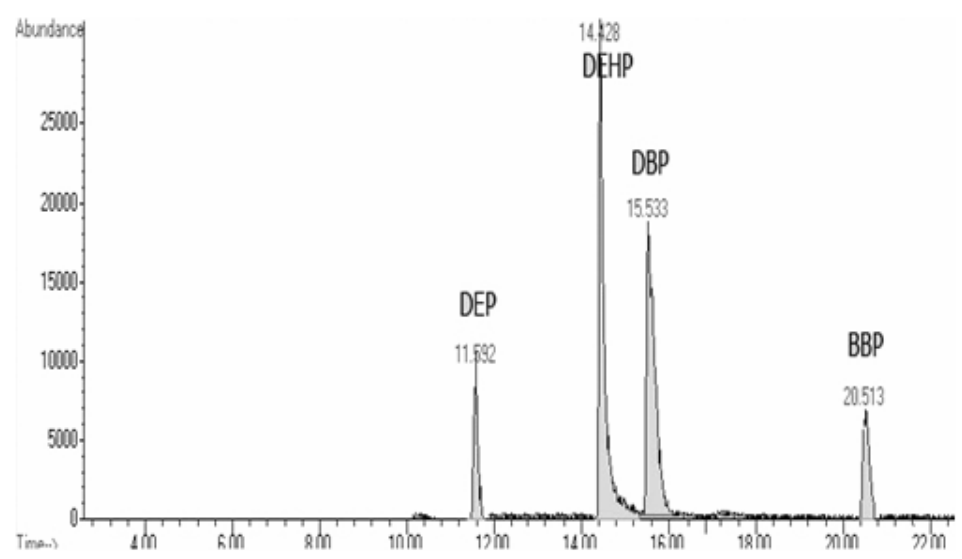

Figure1. A typical chromatogram of di-n-butyl phthalate (DBP), diethyl phthalate (DEP) and Di-(2ethylhexyl) phthalate (DEHP obtained by the method described in material and method section. 


\section{Pes Levels in Infusion Fluids}

Different levels of various phthalate esters were detected in infusion fluids samples. The results are presented in Table2. As seen, the values have relatively high standard deviations due to significant deference in phthalate levels in samples. Various levels of DEHP were detected in all samples (mean \pm SD: $10.55 \pm 6.88 \mathrm{ppb})$ and its highest levels $(19.7 \pm 23.7 \mathrm{ppb})$ were detected in Brand\#1' ringers. Mean total phthalate ester (TPE) levels in infusion fluids were 7.92 \pm 5.68 ppb. $25.1 \pm 17.7 \mathrm{ppb}$ of DEP and $15.4 \pm 8.3 \mathrm{ppb}$ of DBF were detected in brand\#4'dextrose and Brand\#2'injection waters, respectively. Mean
BBP levels in Brand\#1' ringers was about $8.1 \mathrm{ppb}$ while in several samples no levels of DEP, DBP and BBP were detected.

Ringer and normal saline serums respectively contained the highest and lowest levels of DEHP. Total phthalate ester (TPE) levels showed no significant differences between ringer, dextrose and injection water but its levels were significantly lower in normal saline serums (Figure 2). Brand\#3 contains lowest levels of DEHP and TPE. DEHP levels in Brand\#2 products were significantly higher than other Brands, but Brand\#4 contains highest levels of TPE (Figure 3).

Table 2. Levels of four phthalate esters in various infusion fluids of different brands (ppb).

\begin{tabular}{|c|c|c|c|c|c|c|c|c|c|c|c|c|c|c|c|c|}
\hline Type & \multicolumn{16}{|c|}{ Ringer } \\
\hline Brad ID \# & \multicolumn{4}{|c|}{$\# 1$} & \multicolumn{4}{|c|}{$\# 2$} & \multicolumn{4}{|c|}{ \#3 } & \multicolumn{4}{|c|}{$\# 4$} \\
\hline Phthalates & $\mathrm{DE}$ & DEH & DB & BB & $\mathrm{DE}$ & $\mathrm{DEH}$ & DB & BB & $\mathrm{DE}$ & DEH & DB & BB & $\mathrm{DE}$ & DEH & $\mathrm{DB}$ & BB \\
\hline & $\mathrm{P}$ & $\mathrm{P}$ & $\mathrm{P}$ & $\mathrm{P}$ & $\mathrm{P}$ & $\mathrm{P}$ & $\mathrm{P}$ & $\mathrm{P}$ & $\mathrm{P}$ & $\mathrm{P}$ & $\mathrm{P}$ & $\mathrm{P}$ & $\mathrm{P}$ & $\mathrm{P}$ & $\mathrm{P}$ & $\mathrm{P}$ \\
\hline Mean & 15.5 & 19.7 & 9.1 & 8.1 & 5.7 & 9.6 & 2.7 & ND & 4.6 & 4.7 & 3.2 & ND & 16.4 & 8.3 & 4.3 & ND \\
\hline SD & 10.2 & 13.7 & 7.2 & 7.2 & 5.2 & 6.9 & 2.1 & ND & 3.2 & 3.6 & 1.9 & ND & 12.3 & 6.2 & 2.3 & ND \\
\hline Type & \multicolumn{16}{|c|}{ Dextrose } \\
\hline Brad ID \# & \multicolumn{4}{|c|}{$\# 1$} & \multicolumn{4}{|c|}{$\# 2$} & \multicolumn{4}{|c|}{ \#3 } & \multicolumn{4}{|c|}{$\# 4$} \\
\hline Phthalates & $\mathrm{DE}$ & DEH & DB & BB & $\mathrm{DE}$ & $\mathrm{DEH}$ & DB & BB & $\mathrm{DE}$ & DEH & DB & BB & $\mathrm{DE}$ & DEH & $\mathrm{DB}$ & BB \\
\hline & $\mathrm{P}$ & $\mathrm{P}$ & $\mathrm{P}$ & $\mathrm{P}$ & $\mathrm{P}$ & $\mathrm{P}$ & $\mathrm{P}$ & $\mathrm{P}$ & $\mathrm{P}$ & $\mathrm{P}$ & $\mathrm{P}$ & $\mathrm{P}$ & $\mathrm{P}$ & $\mathrm{P}$ & $\mathrm{P}$ & $\mathrm{P}$ \\
\hline Mean & ND & 13.0 & 2.1 & 1.4 & 11.9 & 18.8 & 1.6 & 2.1 & ND & 6.3 & 4.4 & ND & 25.1 & 14.5 & 5.9 & ND \\
\hline SD & ND & 11.8 & 1.7 & 0.8 & 9.3 & 12.9 & 1.1 & 1.5 & ND & 4.5 & 2.4 & ND & 17.7 & 12.7 & 4.1 & ND \\
\hline Type & \multicolumn{16}{|c|}{ Normal saline } \\
\hline Brad ID \# & \multicolumn{4}{|c|}{$\# 1$} & \multicolumn{4}{|c|}{$\# 2$} & \multicolumn{4}{|c|}{ \#3 } & \multicolumn{4}{|c|}{ \#4 } \\
\hline Phthalates & $\mathrm{DE}$ & DEH & DB & $\mathrm{BB}$ & $\mathrm{DE}$ & DEH & DB & BB & $\mathrm{DE}$ & DEH & DB & BB & $\mathrm{DE}$ & DEH & $\mathrm{DB}$ & BB \\
\hline & $P$ & $\mathrm{P}$ & $\mathrm{P}$ & $\mathrm{P}$ & $P$ & $P$ & $P$ & $P$ & $P$ & $\mathrm{P}$ & $\mathrm{P}$ & $\mathrm{P}$ & $\mathrm{P}$ & $\mathrm{P}$ & $P$ & $P$ \\
\hline Mean & ND & 5.2 & 1.8 & 4.7 & 12.1 & 7.8 & 2 & 5.3 & ND & 7.4 & 4.6 & 4.5 & 5.2 & 2.8 & 4.7 & ND \\
\hline SD & ND & 3.8 & 1 & 3.8 & 8.6 & 5.1 & 1.5 & 3.2 & ND & 5.8 & 2.4 & 4.8 & 3.1 & 1.6 & 3.4 & ND \\
\hline Type & \multicolumn{16}{|c|}{ Injection water } \\
\hline Brad ID \# & \multicolumn{4}{|c|}{$\# 1$} & \multicolumn{4}{|c|}{$\# 2$} & \multicolumn{4}{|c|}{ \#3 } & \multicolumn{4}{|c|}{ \#4 } \\
\hline Phthalates & $\mathrm{DE}$ & DEH & DB & BB & $\mathrm{DE}$ & $\mathrm{DEH}$ & DB & BB & $\mathrm{DE}$ & DEH & DB & BB & $\mathrm{DE}$ & DEH & DB & BB \\
\hline & $\mathrm{P}$ & $P$ & $\mathrm{P}$ & $\mathrm{P}$ & $\mathrm{P}$ & $\mathrm{P}$ & $\mathrm{P}$ & $\mathrm{P}$ & $\mathrm{P}$ & $\mathrm{P}$ & $\mathrm{P}$ & $\mathrm{P}$ & $\mathrm{P}$ & $\mathrm{P}$ & $\mathrm{P}$ & $\mathrm{P}$ \\
\hline Mean & ND & 1.9 & 1.4 & ND & 24.1 & 14.8 & 15.4 & 3.8 & 6.5 & 7.5 & 2 & 7.3 & NA & NA & NA & NA \\
\hline SD & ND & 1.5 & 0.8 & ND & 12.3 & 8.8 & 8.3 & 2.3 & 4 & 4.3 & 1.1 & 5.6 & NA & NA & NA & NA \\
\hline
\end{tabular}

ND: Not Detected;

NA: Not Available

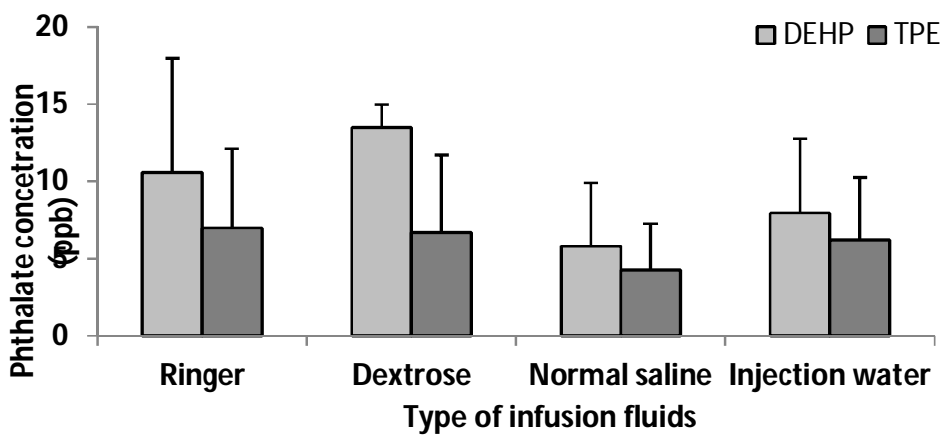

Figure 2. DEHP and TPE (total phthalate esters) levels in different types of infusion fluids (ppb). Data are presented as mean \pm SD. *Significantly different from other types of infusion fluids $(P<0.05)$. 


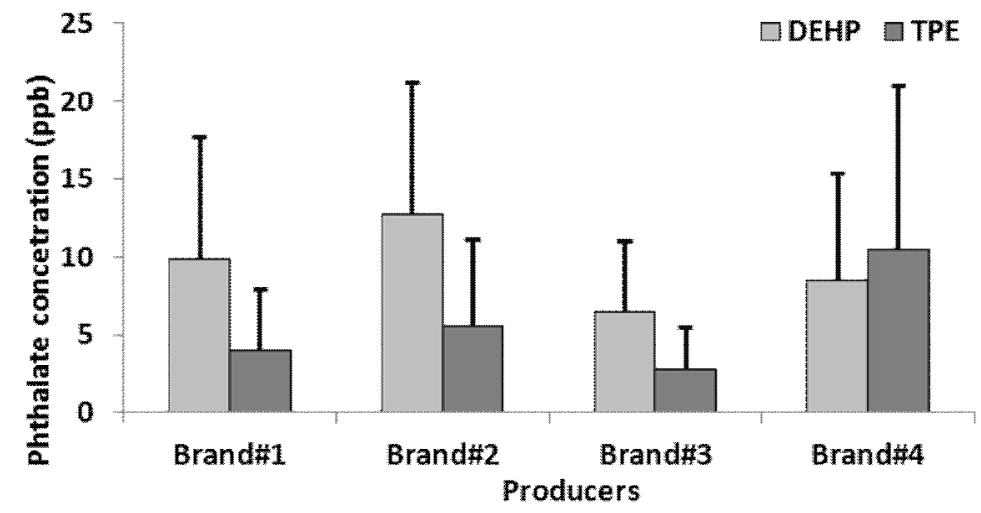

Figure 3. DEHP and TPE levels in infusion fluids (ppb) produced by different pharmaceutical brands. Data are presented as mean $\pm \mathrm{SD}$.

\section{DISCUSSION}

Due to several reports about toxic effects of phthalate esters in animals and humans, most health authorities in different countries have indicated that monitoring of phthalate exposure and related risk assessments require immediate attention [10].

Phthalates are toxic for several body systems. Continues exposure to low doses of these substances showed reproductive toxicity. DEHP and DBP are the most potent reproductive toxicant among the phthalates. Phthalates have great endocrine disruption potency. Phthalates may be responsible for cancer in humans. Asthma and autism also were attributed to phthalate exposure $[9,13]$.

Almost all people are exposed to trace levels of DEHP and other phthalate esters in everyday life via different sources such as food, drinking water and hygiene products (soap, shampoo,etc.)[3]. It is estimated that median DEHP uptake for adult population was 0.71 $\mu \mathrm{g} / \mathrm{kg} /$ day [14] but some condition could seriously enhance phthalate exposure. Tolerable daily intakes (TDI) have been specified by the European Food Safety Authority (EFSA) for several phthalates, and they are $10,500,50 \mu \mathrm{g} / \mathrm{kg}$ bw/day for DBP (EFSA 2005a), BBP (EFSA 2005b), DEHP (EFSA 2005c), respectively [15]. Agency for toxic substances and disease registry (ATSDR) set a maximum residue limit (MRL) level of $7 \mu \mathrm{g} / \mathrm{kg}$ bw/day for DEP [16].

Phthalate release from plastic container into drug solutions in varying degrees [17]. Maximum level of human exposure to DEHP released from blood bags is $0.7 \mathrm{mg} / \mathrm{kg}$ bw/time [4]. Humans undergoing intensive therapeutic interventions are exposed to higher levels of DEHP than the general population [18]. Medications could be a potential source of human exposure to phthalates and urinary levels of DBP metabolites of patient who received asacol [active ingredient mesalamine (mesalazine)] for the treatment of ulcerative colitis were two orders of magnitude higher than the 95th percentile for normal population [19].

Children are a highly sensitive population with increased risk for toxic effects of phthalate esters. The release of phthalate esters from plastic medical devices and pharmaceutical product containers has been the most dangerous for infants because of their small body size, their physical condition and the multiple routes of exposure. Neonates in the Neonatal Intensive CareUnit (NICU) environment constitute a population at particularly increased risk. This is of particular relevance, as these newborns may have been likely previously exposed to phthalate esters in utero[18, 20]

In the present study, various levels of DEHP were detected in all the infusion fluids samples indicating that all pharmaceutical companies use this phthalate ester as a plasticizer in PET container and is leached into the pharmaceutical products. Its average level in different infusion fluids was about $10 \mathrm{ppb}$. This means that each 1liter serums contain ten $\mu \mathrm{g}$ DEHP. These levels of DEHP along with other routes of exposures could have dangerous consequences for patients especially for those that regularly take serumtherapy (because of certain diseases). Comparison of different types of infusion fluids and various brands demonstrated that matrix of infusion fluid is a determining factor and leaching of DEHP and another phthalate ester in into normal saline were significantly lower than other types of serums. 
Further studies can clear the exact mechanisms that by which matrix could influence phthalate leachate. Brand was the other determining factor in phthalate esters levels in infusion fluids and phthalate levels in Brand\#3 were significantly lower than other Bread confirms that pharmaceutical companies use different formulas for the production of these plastic containers and some of them leach lower amounts of phthalates.

\section{CONCLUSION}

This is the first report on leaching of phthalate ester plasticizers into infusion fluids stored in PET containers, and the findings could be useful for the risk assessment of DEHP and other phthalate ester released from plastic containers into pharmaceutical products. We recommend similar studies on other pharmaceutical companies' products. It seems that related regulatory institutions in all countries must monitor these products and encourage pharmaceutical companies to use safer materials for the production of the pharmaceutical plastic container.

\section{ACKNOWLEDGMENT}

This study was supported by a grant from Environment Research Center, Research Institute for Primordial Prevention of Non-communicable Disease, Isfahan University of Medical Sciences, Isfahan, Iran (Grant ID:294179). The authors declare that there is no conflict of interest.

\section{REFERENCES}

1. Shea KM, Health CoE. Pediatric exposure and potential toxicity of phthalate plasticizers. Pediatr 2003;111(6):1467-74.

2. Lorz PM, Towae FK, Enke W, Jäckh R, Bhargava N, Hillesheim W. Phthalic Acid and Derivatives. Ullmann's Encyclopedia of Industrial Chemistry: Wiley-VCH Verlag GmbH \& Co. KGaA; 2000.

3. Latini G. Monitoring phthalate exposure in humans. Clin Chim Acta 2005;361(1):20-9.

4. Inoue K, Kawaguchi M, Yamanaka R, Higuchi T, Ito R, Saito K, et al. Evaluation and analysis of exposure levels of di (2-ethylhexyl) phthalate from blood bags. Clin Chim Acta 2005;358(1):159-66.

5. Glue C, Platzer MH, Larsen ST, Nielsen GD, Skov PS, Poulsen LK. Phthalates potentiate the response of allergic effector cells. Basic Clin Pharmacol Toxicol 2005;96(2):140-2.

6. Schettler T. Human exposure to phthalates via consumer products. Int J Androl 2006;29(1):134-9.
7. Lyche JL, Gutleb AC, Bergman $\AA$, Eriksen GS, Murk AJ, Ropstad E, et al. Reproductive and developmental toxicity of phthalates. J Toxicol Environ Health BCrit Rev 2009;12(4):225-49.

8. Martino-Andrade AJ, Chahoud I. Reproductive toxicity of phthalate esters. Mol Nutr Food Res 2010;54(1):148-57.

9. Chen X, Xu S, Tan T, Lee ST, Cheng SH, Lee FWF, et al. Toxicity and estrogenic endocrine disrupting activity of phthalates and their mixtures. Int J Environ Res Public Health 2014;11(3):315668.

10. Ventrice P, Ventrice D, Russo E, De Sarro G. Phthalates: European regulation, chemistry, pharmacokinetic and related toxicity. Environ Toxicol Pharmacol 2013;36(1):88-96.

11. Liang $\mathrm{P}, \mathrm{Xu} \mathrm{J}, \mathrm{Li} \mathrm{Q}$. Application of dispersive liquid-liquid microextraction and highperformance liquid chromatography for the determination of three phthalate esters in water samples. Anal Chim Acta 2008;609(1):53-8.

12. Ermer J, Miller JHM. Method validation in pharmaceutical analysis: A guide to best practice: John Wiley \& Sons; 2006.

13. Mankidy R, Wiseman S, Ma H, Giesy JP. Biological impact of phthalates. Toxicol Lett 2013;217(1):50-8.

14. Blount BC, Silva MJ, Caudill SP, Needham LL, Pirkle JL, Sampson EJ, et al. Levels of seven urinary phthalate metabolites in a human reference population. Environ Health Perspect 2000;108(10):979-80.

15. Cao XL. Phthalate esters in foods: sources, occurrence, and analytical methods. Comprehensive Compr Rev Food Sci Food Saf 2010;9(1):21-43.

16. Heudorf U, Mersch-Sundermann V, Angerer J. Phthalates: toxicology and exposure Int $\mathrm{J}$ Hyg Environ Health 2007;210(5):623-34.

17. Inoue $\mathrm{K}$, Higuchi $\mathrm{T}$, Okada $\mathrm{F}$, Iguchi $\mathrm{H}$, Yoshimura Y, Sato A, et al. The validation of column-switching LC/MS as a high-throughput approach for direct analysis of di (2-ethylhexyl) phthalate released from PVC medical devices in intravenous solution. $\mathrm{J}$ Pharm Biomed Anal 2003;31(6):1145-52.

18. Calafat AM, Needham LL, Silva MJ, Lambert G. Exposure to di-(2-ethylhexyl) phthalate among premature neonates in a neonatal intensive care unit. Pediatrics 2004;113(5):e429-e34.

19. Hernández-Díaz S, Su Y-C, Mitchell AA, Kelley KE, Calafat AM, Hauser R. Medications as a potential source of exposure to phthalates among women of childbearing age. Reprod Toxicol 2013;37:1-5.

20. Latini G. Potential hazards of exposure to di-(2ethylhexyl)-phthalate in babies. Neonatology 2000;78(4):269-76. 